\section{LEVETIRACETAM MONITORING IN BREAST-MILK: HIGH INTER-INDIVIDUAL VARIABILITY}

${ }^{1} \mathrm{~N}$ Dinavitser ${ }^{*},{ }^{1} \mathrm{E}$ Kohn, ${ }^{1} \mathrm{M}$ Berlin, ${ }^{2,3} \mathrm{~A}$ Livne, ${ }^{2,3} \mathrm{R}$ Keidar, ${ }^{4} \mathrm{~L}$ Ben-Nun, ${ }^{4} \mathrm{~N}$ Agajani, ${ }^{4} \mathrm{R}$ Sheinberg, ${ }^{4} \mathrm{~S}$ Gamsu, ${ }^{4} \mathrm{H}$ Flor-Hirsch, ${ }^{5} \mathrm{~N}$ Brandriss, ${ }^{5} \mathrm{~A}$ Bar-Chaim, ${ }^{6} \mathrm{D}$ Stepenski, ${ }^{1,3} \mathrm{M}$ Berkovitch. ${ }^{1}$ Clinical Pharmacology and Toxicology; ${ }^{2}$ Department of Neonatology, Assaf Harofeh Medical Centre, Zerifin; ${ }^{3}$ Sackler Faculty of Medicine, Tel-Aviv University, Tel Aviv; ${ }^{4}$ Division of Pediatrics; ${ }^{5}$ Laboratories Department, Biochemistry lab, Assaf Harofeh Medical Centre, Zerifin; ' ${ }^{6}$ Pharmacy Faculty, Ben Gurion University of the Negev, Beer Sheva, Israel

\subsection{6/archdischild-2019-esdppp.31}

Background Anti-epileptic drug therapy is a great challenge for the practitioners during pregnancy and lactation. Levetiracetam (LEV) is commonly prescribed to pregnant women, however, there are only few publications on its use during lactation with small number of participants.

Objective To monitor LEV levels in breast-milk of epileptic mothers treated with LEV.

Methods Breastfeeding women treated with LEV during pregnancy and after delivery were recruited. Milk sample was collected before administration of the drug and other samples were collected at time points of 1,3,6,9, and 12 hours after drug administration. Breastmilk and blood LEV levels were measured using HPLC.

Results Fourteen breastfeeding women participated in the study: 9 infants were fully breastfed whereas 5 were partially breastfed. Maternal average daily dose of LEV was $2517 \mathrm{mg}$. Average infant's age was 8 weeks (3-22w). Average infant's weight 4368 gr (3300-7000 gr). Milk/Plasma LEV concentration ratio was 0.88 (0.23-1.1). Relative Infant Dose (RID) was $40 \%$ in partial breast feeding, and $61 \%$ in full breastfeeding. Estimated average daily dose that all infants received through milk was $158 \mathrm{mg} / \mathrm{d}$ (83-250 mg). The normalized dose for the average infant weight per day was $36 \mathrm{mg}$, which is $15 \%$ less than the maximal daily dose of LEV in infants (max. daily dose in infants 1-6 months in $42 \mathrm{mg} / \mathrm{d}$ ). No adverse reactions were observed in the breastfeed infants.

Conclusions Although the RID of LEV were found to be high, no adverse reactions were observed in the infants; Nevertheless, further studies are needed to elucidate the high variability of LEV excretion into breastmilk.

Disclosure(s) Nothing to disclose

\section{ONTOGENY OF HUMAN KIDNEY OCT2 EXPRESSION ACROSS THE PAEDIATRIC AGE RANGE}

${ }^{1} \mathrm{~N}$ Smeets*, ${ }^{2} B$ van Groen, ${ }^{1} J$ Pertijs, ${ }^{1} M$ Wilmer, ${ }^{3} B$ Smeets, ${ }^{4} R$ Verdijk, ${ }^{1,2} S$ de Wildt. ${ }^{1}$ Pharmacology and Toxicology, Radboudumc, Nijmegen; ${ }^{2}$ Intensive Care and Pediatric Surgery, Erasmus MC - Sophia Childrens Hospital, Rotterdam; ${ }^{3}$ Pathology, Radboudumc, Nijmegen; ${ }^{4}$ Pathology, Erasmus MC, Rotterdam, The Netherlands

\subsection{6/archdischild-2019-esdppp.32}

Background In adults, the organic cation transporter 2 (protein name OCT2, gene name SLC22A2) is localised in the kidney proximal tubules where it mediates organic cation secretion. Hence, the transporter plays a role in the disposition and excretion of several drugs and drug-drug interactions. To better understand the disposition of OCT2 substrate drugs in children, we studied OCT2 localisation and expression in paediatric kidney tissue.

Methods The expression of OCT2 was visualised in tissue using immunohistochemical staining. Tissues were derived post-mortem from children aged $0-14$ years. Gestational age varied between 24 and 40 weeks. Intensity of the staining at the basolateral membrane was scored by two individual observers using three categories; negative, detectible and high. Agreement between two observers was determined using Cohen's kappa.

Results 44 kidney samples $(n=17$ neonates, $n=17$ infants, $\mathrm{n}=7$ children, $\mathrm{n}=3$ adolescent) were analysed and scored There was substantial agreement between two judgements with a kappa of $0.773(\mathrm{p}<0.005)$. No age related pattern was observed in the expression of OCT2. Even in the youngest age group, the expression of OCT2 was clearly visible.

Conclusion The kidney expression of OCT2 did not show an age-related pattern. In all age groups, expression levels were similar and OCT2 was properly localised at the basolateral membrane. These findings suggest that, with increasing age, OCT2 will not influence the renal excretion of its substrates. Disclosure(s) Nothing to disclose

\section{TOWARDS PERSONALISED WEANING OF SEDATIVES AND ANALGESICS USING MECHANISM-BASED MODELLING OF IATROGENIC WITHDRAWAL IN CRITICALLY ILL CHILDREN}

${ }^{1} \mathrm{~S}$ Goulooze*, ${ }^{1} \mathrm{E}$ Krekels, ${ }^{2,3} \mathrm{M}$ van Dijk, ${ }^{1} \mathrm{~T}$ Hankemeier, ${ }^{2} \mathrm{D}$ Tibboel, ${ }^{2} \mathrm{E}$ Ista, ${ }^{1,4} \mathrm{C}$ Knibbe. ${ }^{1}$ Department of Systems Biomedicine and Pharmacology, Leiden Academic Centre for Drug Research, Leiden University, Leiden; ${ }^{2}$ Intensive Care and Pediatric Surgery; ${ }^{3}$ Division of Neonatology, Erasmus Medical Center-Sophia Children's Hospital, Rotterdam; ${ }^{4}$ Department of Clinical Pharmacy, St. Antonius Hospital, Nieuwegein, The Netherlands

\subsection{6/archdischild-2019-esdppp.33}

Background Prolonged treatment with analgesics and sedatives can result in iatrogenic withdrawal syndrome (IWS) in children being weaned from these drugs. ${ }^{1}$ Personalized weaning strategies might lower the incidence of IWS, but this requires a quantitative understanding of withdrawal over time in individual patients.

Methods Data from 81 children (aged 1 month to 17 years) collected during an observational clinical study on IWS $^{2}$ were used, including a total of 1782 withdrawal assessments performed by PICU nurses, on a numerical rating scale $\left(\mathrm{NRS}_{\text {with- }}\right.$ drawal) from 0 (no withdrawal) to 10 (worst withdrawal possible). Population pharmacokinetic models from literature were used to generate concentration-time profiles in each patient of all key analgesics and sedatives: morphine, fentanyl, methadone, midazolam, lorazepam, propofol, esketamine and clonidine. A mechanism-based withdrawal model was developed using NONMEM 7.3 to quantify IWS over time. The final model was used to perform simulations in which different weaning strategies were compared.

Results A novel mechanism-based withdrawal model structure was developed with a hypothetical compartment, which equilibrates with the central pharmacokinetic compartment, and which characterizes the development and disappearance of drug dependence over time. With this model and available data, withdrawal dynamics could be established with statistical significance for fentanyl $\left(\mathrm{p}<10^{-6}\right)$, morphine $(\mathrm{p}=0.043)$ and esketamine $(p=0.002)$, and not for any of the other drugs. Compared with fentanyl, development and disappearance of esketamine and morphine dependence is slower.

Conclusions Given the patient's use of fentanyl, morphine and esketamine, the developed model can dynamically predict IWS from these substances under different weaning strategies. The results show that the optimal strategy for weaning of drug 